\title{
Upper Bounds to Transport Capacity of Wireless Networks
}

\author{
Aleksandar Jovičić Student Member, IEEE, Pramod Viswanath, Member, IEEE \\ Sanjeev R. Kulkarni, Fellow, IEEE
}

\begin{abstract}
We derive upper bounds on the transport capacity of wireless networks. The bounds obtained are solely dependent on the geographic locations and power constraints of the nodes. As a result of this derivation, we are able to conclude the optimality, in the sense of scaling of transport capacity with the number of nodes, of a multi-hop communication strategy for a class of network topologies.
\end{abstract}

Index Terms-Ad-hoc wireless networks, cut-set bounds, multi-hop, transport capacity

\section{INTRODUCTION}

$\mathbf{U}$ NDERSTANDING network communication has remained the holy grail of information theory; even the simplest settings have largely uncharacterized capacity regions. Recently, some progress in the study of wireless networks has been made by asking questions coarser than precise achievable rate regions. In particular, the seminal work of [4] introduced the measure transport capacity: The total bitmeters per second a network can reliably support. Further, the authors studied the rate of growth of this performance measure as a function of the network resource- the number of radios (equivalently, nodes) themselves. The key result of [4] is to identify the rate of growth of transport capacity of an $n$ radio network using a simple nearest neighbor multihop communication scheme.

In the multihop communication scheme we treat simultaneous transmissions as interference, and so a successful transmission over a distance $d$ means that there are no other transmissions in an area proportional to $d^{2}$. There is now a (potential) tradeoff between a need to communicate long distances and allowing many simultaneous transmissions. In a regular (or close to regular) network it is optimal to always communicate to nearest neighbors. With this, the transport capacity of regular minimum distance networks grows linearly in $n$. It is not clear how much we are missing out by focusing on a simple multihop store-and-forward communication strategy.

In irregular networks there is crowding of nodes and the transport capacity with multihop communication is reduced;

Manuscript received November, 2003; revised March, 2004. This research was supported in part by the Army Research Office under grant DAAD19-001-0466, Draper Laboratory under IR\&D 6002 grant DL-H-546263, and the National Science Foundation under grants CCR-0312413, CCR-0237549, and a grant from Motorola Inc., as part of the Motorola Center for Communications. An earlier version of the results of this paper was presented at the IEEE International Symposium on Information Theory, Yokohoma, Japan, 2003.

A. Jovičić and P. Viswanath are with the department of Electrical and Computer Engineering at the University of Illinois at Urbana-Champaign. S. R. Kulkarni is with the Electrical Engineering department at Princeton University. this is studied in a general framework in [6]. Again, it is not clear if this reduction is due to our choice of the communication strategy (multihop) or a fundamental property of the location of the nodes. The goal of this paper is to provide an answer to these questions by deriving some simple fundamental upper bounds to the transport capacity. The bounds we provide are solely a function of network topology, i.e., the geographic locations of the nodes. As a by-product of this derivation, we will be able to conclude the optimality of multihop communication for a large class of networks (optimality in the sense of the same rate of growth with the number of nodes). This is a strengthening of the same conclusion arrived at in [12], which also studied the performance of other non-multihop communication schemes.

We study the following general wireless network: The received signal at node $j$ at (slotted) time $m$ is

$$
y_{j}[m]=\sum_{i \neq j} h_{i j}[m] x_{i}[m]+z_{j}[m] .
$$

Here $x_{i}[m]$ is the signal transmitted by node $i$ at time $m$ and $z_{j}[m]$ is i.i.d. white Gaussian noise. The multiplier $h_{i j}[m]$ is defined as

$$
h_{i j}[m]:=\frac{h_{i j}^{s}[m]}{\left(1+r_{i j}\right)^{\delta}},
$$

where $\left\{h_{i j}^{s}[m]\right\}_{m}$, a stationary and ergodic stochastic process that is independent for each pair of nodes $(i, j)$, models the small scale fluctuations of frequency flat fading. For simplicity we assume that $\mathbb{E}\left[\left|h_{i j}^{s}[m]\right|^{2}\right]=1$ for all $i, j, m$. The large scale variations are modeled explicitly through the decay of signal level: A factor of $\frac{1}{\left(1+r_{i j}\right)^{\delta}}$ from node $i$ to node $j$, with $r_{i j}$ denoting the (Euclidean) distance between the nodes $i$ and $j$. The parameter $\delta$ is the rate of signal decay, ${ }^{1}$ which is unity in free space, two with a single reflected path along with the line of sight, and possibly greater than 2.5 in crowded urban environments [3].

We are interested in peer-peer communication, i.e., the nodes are communicating among themselves. The focus of this paper is to derive information theoretic upper bounds to transport capacity, which is defined as

$$
C_{T}:=\sup \sum_{i, j=1}^{n} R_{i j} r_{i j}
$$

\footnotetext{
${ }^{1}$ The far field signal decay is usually denoted by $r_{i j}^{-\delta}$. Here we have written $\left(1+r_{i j}\right)^{-\delta}$ to ensure that our model makes sense when nodes get close; i.e., the average received power is not more than the average transmit power.
} 
where the supremum is taken over all the feasible $n \times n$ rate matrices $\left[R_{i j}\right]$ (a rate matrix $\left[R_{i j}\right]$ is feasible if simultaneous reliable communication at rates $R_{i j}$ is possible, for all nodes $i$ and $j$ ).

The only general upper bound in network information theory is the cut-set bound (Theorem 4 in [1], Theorem 14.10.1 in [2]). Our main result is to use a random cut-set to derive the following upper bound on transport capacity. We consider two (disparate) models of the wireless fading channel. If the channel variations are "slow" enough so that coherent communication is possible, we model this scenario by allowing full knowledge at each node of the fading channels from all the other nodes. This is also known as the full CSI scenario (channel state information at both the transmitter and receiver nodes). In the scenario where the channel is changing rapidly enough so that coherent communication is not feasible, our model is to assume complete ignorance of the channel realizations by all the nodes. This is the no CSI model. Clearly these are two extreme models but they serve to cover the ranges of channel fluctuations and allow us to make simple statements about the network communication problem. Each node $j$ has an average transmit power constraint (denoted by $P_{j}$.

Main Result: The transport capacity is upper bounded by

$$
\sum_{i, k, j=1}^{n} \frac{\sqrt{P_{i} P_{k}} \min \left(r_{i j}, r_{k j}\right)}{\left(1+r_{i j}\right)^{\delta}\left(1+r_{k j}\right)^{\delta}} .
$$

With no channel state information at the nodes, we can sharpen the upper bound to

$$
\sum_{i, j=1}^{n} \frac{P_{i} r_{i j}}{\left(1+r_{i j}\right)^{2 \delta}}
$$

This upper bound on the transport capacity is entirely in terms of the geographic locations and the power constraints of the nodes. We see that the upper bound is quite robust to "small" (in a network sense) fluctuations of the geographic locations of the nodes. For example, removing one node from the network does not alter the performance by much. Or if two specific nodes were moved very close to each other, then again the upper bound does not change by much.

This upper bound allows us to study scaling laws (rate of growth of transport capacity with number of nodes) for specific network topologies. Consider minimum distance networks (with a minimum distance of, say $r_{\min }>0$, between any two nodes) on a line and on a plane (a scenario studied in [12]).

1) The transport capacity of minimum distance networks on a line is upper bounded by $c_{1} n$ as long as $\delta>1.5$. With no channel state information the same bound holds with $\delta>1$.

2) The transport capacity of minimum distance networks on a plane is upper bounded by $c_{2} n$ as long as $\delta>2.5$. With no channel state information the same bound holds with $\delta>1.5$.

Here $c_{1}$ and $c_{2}$ are constants that depend on the actual node locations; simple upper bounds to these constants are derived in Section III. In a recent (and independent) work [13], the authors show that a transport capacity of $c_{0} n$ is achievable via multihop communication in wireless networks with the timevarying fading model expressed in (1) (see Theorem 3.2 of [13]). Hence, we can conclude that multihop communication is optimal in the sense of scaling of transport capacity with the number of nodes. This is a strengthening of the same result in [12], which considered a simpler, time-invariant channel model and required stronger conditions on $\delta$. The essence of our derivation is to argue that even when nodes team up (at the transmitter side and at the receiver side) the spatial separation prevents them from forming many parallel channels (in other words, the spatial degrees of freedom are limited). A result similar in spirit is arrived at independently in [7] for the sumcapacity of the network, under a time-invariant channel model.

We can use our upper bound to study random networks and show the robustness of this scaling law optimality of multihop communication. In particular, when the nodes are randomly and uniformly placed in an area growing linearly with the number of nodes (on a line, the overall length is of order $n$ ), the transport capacity is upper bounded by $O(n \log n)$ for almost every realization of node locations with no CSI at the nodes; with full CSI at the nodes, the upper bound is $O\left(n(\log n)^{2}\right)$. On the other hand, multihop communication can achieve a transport capacity of order $\frac{n}{\sqrt{\log n}}$ for almost every realization of node locations (Section 3 of [6]). In comparison to the dominant term (linear growth in $n$ ) the logarithmic factors are small, which shows the near-optimality of multihop communication in this context of random node locations.

Upon a closer look at the upper bound for transport capacity in (3) and (4), we see the linearity of transport capacity in the transmit power. Communication in a large network is primarily interference-limited and occurs at low SNRs (signal to noise ratios); in this regime, linearity of SNR w.r.t. the communication rate is a good approximation. If the nodes have widely different power capabilities and this capability can be tuned to specific network topologies, then the statement of our main result may be too weak. To understand the role of power constraints on transport capacity, we consider the following scenario: there is only an overall transmit power constraint (growing linearly with the number of nodes) and this can be distributed among the nodes in any manner (that is suited to the specific network topology). In this case, it turns out that the number of spatial degrees of freedom that cooperation between transmitting and receiving nodes can harness is approximately proportional to $n^{\frac{1}{2 \delta}}$. This statement can be made precise in the context of minimum distance networks on both a line and a plane. Minimum distance networks with an overall power constraint have a transport capacity upper bounded by $c_{3} n^{1+\frac{1}{2 \delta-1}} \log n$. If the nodes have no channel state information, then we can sharpen this bound to $c_{4} n^{1+\frac{1}{2 \delta}} \log n$. We have the same conditions on the decay rate $\delta$ as in the individual power constraint scenario mentioned earlier. Simple upper bounds to $c_{3}$ and $c_{4}$ are derived in Section V.

The main result is derived in Section $I I$. The scaling laws for minimum distance networks are studied in Section III. 
The role of random node locations is studied in Section $I V$. We conclude with a discussion of the import of our results and list some open questions.

\section{MAin Result}

Suppose there are $n$ nodes in the network $\mathcal{N}$ and each node is power-limited to $P_{i}, i \in \mathcal{N}$. Let $r_{i j}$ denote the distance between the nodes $i$ and $j$ for $i, j \in \mathcal{N}$. Suppose now that we divide the nodes by partitioning $\mathcal{N}$ into complementary sets $\mathcal{C}^{+}$and $\mathcal{C}^{-}$with cardinalities $n_{\mathcal{C}^{+}}$and $n_{\mathcal{C}^{-}}$. As stated in the introduction, our main result is the following theorem.

Theorem 2.1: Consider an arbitrary configuration of $n$ nodes in the plane. With no channel state information (CSI) at the nodes, the transport capacity is upper bounded by

$$
C_{T} \leq \sum_{i, j \neq i} P_{i} \frac{r_{i j}}{\left(1+r_{i j}\right)^{2 \delta}}
$$

With full CSI at the nodes, the transport capacity is further upper bounded by

$$
C_{T} \leq \sum_{i, k, j \neq i, k} \sqrt{P_{i} P_{k}} \frac{\min \left(r_{i j}, r_{k j}\right)}{\left(1+r_{i j}\right)^{\delta}\left(1+r_{k j}\right)^{\delta}} .
$$

Proof: There are two key ideas used in the proof:

1) We can bound the sum-rate of communication across a given cut by the capacity of a point-to-point, fading AWGN channel with $n_{\mathcal{C}}+$ transmit and $n_{\mathcal{C}-}$ receive antennas.

2) We can use a random straight line to geographically partition the nodes and then average the bound over the distribution of this line to arrive at a bound on transport capacity.

We begin with the no CSI model. Now suppose that after we partition $\mathcal{N}$ into $\mathcal{C}^{+}$and $\mathcal{C}^{-}$, a genie provides CSI to only the nodes in $\mathcal{C}^{-}$. Clearly, more information can only result in a larger rate. The assumption that only the nodes in $\mathcal{C}^{-}$know the channel realizations is equivalent to the assumption of receiver-only CSI in a MIMO (multiple input, multiple output)

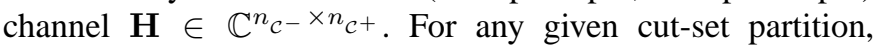
the sum rate of communication across the cut is then upper bounded by the capacity of such a MIMO channel:

$$
\begin{aligned}
\sum_{i \in \mathcal{C}^{+}, j \in \mathcal{C}^{-}} R_{i j} \leq \max _{\substack{\mathbf{K}_{x} \succeq 0: \\
\left(\mathbf{K}_{x}\right)_{i i} \leq P_{i}}} \mathbb{E}\left[\log \operatorname{det}\left(\mathbf{I}+\mathbf{H K}_{x} \mathbf{H}^{\dagger}\right)\right] \\
:=\max _{\mathbf{K}_{x} \succeq 0:\left(\mathbf{K}_{x}\right)_{i i} \leq P_{i}} R\left(\mathbf{K}_{x}\right) .
\end{aligned}
$$

Here we have normalized the variance of the background additive white Gaussian noise to unity. If we model the processes $\left\{h_{i j}[m]\right\}$ as independent and the stationary distribution to be symmetric around the origin, then one can show that the optimal covariance matrix is diagonal, i.e., the maximum in (7) is attained with $\left(\mathbf{K}_{x}\right)_{k k}=P_{i_{k}}$ for $k=1,2, \ldots, n_{\mathcal{C}^{+}}$. This result has been shown in somewhat different ways in the literature [5], [9], [10], [11]. We provide a simple proof in the appendix for completeness (expressed as (35)). Evaluating (7) for this diagonal $\mathbf{K}_{x}$ and using the fact that the determinant of a Hermitian matrix is at most as large as the product of

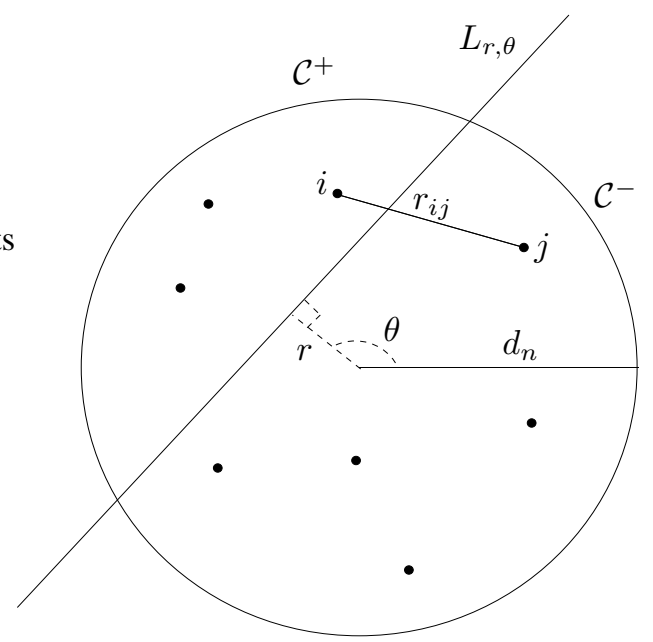

Fig. 1. Uniformly random line $L_{r, \theta}$ partitioning the $n$ node network.

its diagonal entries (the Hadamard inequality), we obtain the following:

$$
\begin{aligned}
\max _{\substack{\mathbf{K}_{x} \succeq 0: \\
\left(\mathbf{K}_{x}\right)_{i i} \leq P_{i}}} R\left(\mathbf{K}_{x}\right) & \leq \mathbb{E}\left[\sum_{j \in \mathcal{C}^{-}} \log \left(1+\sum_{i \in \mathcal{C}^{+}} P_{i}\left|h_{i j}\right|^{2}\right)\right] \\
& \leq \sum_{j \in \mathcal{C}^{-}} \log \left(1+\sum_{i \in \mathcal{C}^{+}} \frac{P_{i}}{\left(1+r_{i j}\right)^{2 \delta}}\right),(8) \\
& \leq \sum_{j \in \mathcal{C}^{-}} \sum_{i \in \mathcal{C}^{+}} \frac{P_{i}}{\left(1+r_{i j}\right)^{2 \delta}} .
\end{aligned}
$$

The last two steps are obtained by applying Jensen's inequality and the fact that $\log (1+x) \leq x$. Now suppose that this partitioning of $\mathcal{N}$ was accomplished by a uniformly random straight line, geographically splitting the network into $\mathrm{C}^{+}$ and $\mathcal{C}^{-}$. Since there is a finite number of nodes, we can circumscribe them by a circle, the radius of which we will denote by $d_{n}$.

Suppose that this random line $L_{r, \theta}$ is parameterized by polar coordinates $(r, \theta)$ which are each independently and uniformly distributed over $\left[0, d_{n}\right]$ and $[0,2 \pi]$, respectively (see Figure 1 ). Then it is easy to show that the probability of a uniformly random line cutting any two nodes $i, j \in \mathcal{N}$ is exactly $\frac{r_{i j}}{\pi d_{n}}$.

This also follows from the following classical result from stochastic geometry (see [8]): Let $A$ be a bounded convex set in $\mathbb{R}^{2}$ and $c \subset A$ be a curve of finite length. Then, the expected number of times a random line intersects $c$ is equal to $2 L(c) / \operatorname{perim}(A)$, where $L(c)$ is the length of the curve $c$ and $\operatorname{perim}(A)$ is the perimeter of the set $A$.

Here $A$ is a circle of radius $d_{n}$ and $c$ is the line joining the nodes $i$ and $j$. This result allows us to express transport capacity (2) as the supremum (over all feasible rate vectors) of the (scaled) average of the sum-rates of communication across all cuts generated by a uniformly random line. If we let $\mathbf{1}_{\{A\}}$ denote the indicator function of the event $A$ (defined to be one if $A$ is true and zero otherwise), and let $\mathbb{E}_{\mathcal{C}}$ denote the expectation operator with respect to the distribution of the cut-sets $\left(\mathcal{C}^{+}, \mathcal{C}^{-}\right)$generated by the uniformly random line, we 
can express this fact in the following way:

$$
\begin{aligned}
\mathbb{E}_{\mathcal{C}}\left[\sum_{i \in \mathcal{C}^{+}, j \in \mathcal{C}^{-}} R_{i j}\right] & =\mathbb{E}_{\mathcal{C}}\left[\sum_{i, j=1}^{n} R_{i j} \mathbf{1}_{\left\{i \in \mathcal{C}^{+}, j \in \mathcal{C}^{-}\right\}}\right] \\
& =\sum_{i, j=1}^{n} R_{i j} \mathbb{P}\left(i \in \mathcal{C}^{+}, j \in \mathcal{C}^{-}\right) \\
& =\sum_{i, j=1}^{n} R_{i j} \frac{r_{i j}}{2 \pi d_{n}} .
\end{aligned}
$$

Here we used the fact that

$$
\begin{aligned}
\mathbb{P}((i, j) \text { cut }) & =\mathbb{P}\left(\left\{i \in \mathcal{C}^{-}, j \in \mathcal{C}^{+}\right\} \cup\left\{i \in \mathcal{C}^{+}, j \in \mathcal{C}^{-}\right\}\right), \\
& =\frac{r_{i j}}{\pi d_{n}}
\end{aligned}
$$

implies that $\mathbb{P}\left(i \in \mathcal{C}^{+}, j \in \mathcal{C}^{-}\right)=\frac{r_{i j}}{2 \pi d_{n}}$ since, given a random line realization, the assignment of $\left(\mathcal{C}^{+}, \mathcal{C}^{-}\right)$is unique up to a permutation, both permutations being equally likely. Scaling (10) by $2 \pi d_{n}$ and taking the supremum over all feasible $n \times n$ rate matrices $\left[R_{i j}\right]$, one arrives at the transport capacity as defined in (2). Thus we can find upper-bounds on $C_{T}$ by first upper-bounding the sum-rate across each cut and then averaging (and scaling) this bound over all cuts. In particular, from (9) we have that

$$
\begin{aligned}
C_{T} & \leq 2 \pi d_{n} \mathbb{E}_{\mathcal{C}}\left[\sum_{j \in \mathcal{C}^{-}} \sum_{i \in \mathcal{C}^{+}} \frac{P_{i}}{\left(1+r_{i j}\right)^{2 \delta}}\right], \\
& =2 \pi d_{n} \sum_{i, j=1}^{n} \frac{P_{i}}{\left(1+r_{i j}\right)^{2 \delta}} \frac{r_{i j}}{2 \pi d_{n}}, \\
& =\sum_{i, j=1}^{n} P_{i} \frac{r_{i j}}{\left(1+r_{i j}\right)^{2 \delta}},
\end{aligned}
$$

thus proving the first part of the theorem.

We now turn to the full CSI model. Here full channel state information is available at both the transmitter and receiver in a MIMO channel. Thus the sum-rate bound on the communication across the cut, for a given partition, now takes the form

$$
\begin{aligned}
\sum_{\substack{i \in \mathcal{C}^{+} \\
j \in \mathcal{C}^{-}}} R_{i j} & \leq \mathbb{E}\left[\max _{\substack{\mathbf{K}_{x} \succeq 0: \\
\left(\mathbf{K}_{x}\right)_{i i} \leq P_{i}}} \log \operatorname{det}\left(\mathbf{I}+\mathbf{H} \mathbf{K}_{x} \mathbf{H}^{\dagger}\right)\right] \\
& \leq \mathbb{E}\left[\sum_{j \in \mathcal{C}^{-}} \max _{\substack{\mathbf{K}_{x} \succeq 0: \\
\left(\mathbf{K}_{x}\right)_{i i} \leq P_{i}}} \log \left(1+\mathbf{h}_{j} \mathbf{K}_{x} \mathbf{h}_{j}^{\dagger}\right)\right]
\end{aligned}
$$

Here $\mathbf{h}_{j}$ is the $j$-th row of $\mathbf{H}$. Every principal minor of a positive semidefinite matrix is nonnegative. In particular if $A$ is a p.s.d. matrix then $a_{i i} a_{j j} \geq\left|a_{i j}\right|^{2}$. We use this fact to get

$$
\begin{aligned}
\max _{\substack{\mathbf{K}_{x} \succeq 0: \\
\left(\mathbf{K}_{x}\right)_{i i} \leq P_{i}}} \mathbf{h}_{j} \mathbf{K}_{x} \mathbf{h}_{j}^{\dagger} & =\max _{\substack{\mathbf{K}_{x} \succeq 0: \\
\left(\mathbf{K}_{x}\right)_{i i} \leq P_{i}}} \sum_{i, k \in \mathcal{C}^{+}} h_{i j}\left(\mathbf{K}_{x}\right)_{i k} h_{k j}^{*}, \\
& \leq \sum_{i, k \in \mathcal{C}^{+}} \sqrt{P_{i} P_{k}}\left|h_{i j} h_{k j}\right| .
\end{aligned}
$$

Substituting this calculation in (11) and using the independence of the small scale variations between different channels (and our normalization of the average amplitude of the small scale variations to unity) we arrive at a bound on the sum-rate:

$$
\sum_{\substack{i \in \mathcal{C}^{+} \\ j \in \mathcal{C}^{-}}} R_{i j} \leq \sum_{j \in \mathcal{C}^{-}} \sum_{i, k \in \mathcal{C}^{+}} \frac{\sqrt{P_{i} P_{k}}}{\left(1+r_{i j}\right)^{\delta}\left(1+r_{k j}\right)^{\delta}}
$$

As before, we average (12) over the cut-sets by observing that the probability that the node $j$ and the pair of nodes $(i, k)$ are cut by a uniformly random line cannot be larger than the probability of cutting either the nodes $j$ and $i$ or nodes $j$ and $k$. We pick the smaller of the two probabilities, $\frac{\min \left(r_{j i}, r_{j k}\right)}{2 \pi d_{n}}$, for the upper bound. Multiplying the resulting average by $2 \pi d_{n}$, we obtain (6), thus proving the theorem.

\section{Scaling Laws For Minimum Distance Networks}

In this section, we look at the applicability of our upper bounds on transport capacity derived in Section II to some specific network topologies. In particular, we consider minimum distance networks, where any pair of nodes is separated by a distance at least $r_{\min }>0$. We consider networks on a line and on the plane. Our main goal is to characterize the rate of growth of the upper bounds to transport capacity as a function of the number of nodes. We denote $P$ as a uniform upper bound to the average transmit power constraints of the radios.

Corollary 3.1: Suppose the nodes lie on a line at a distance of at least $r_{\min }>0$ from each other. With no CSI at the nodes, the transport capacity is upper bounded by

$$
\frac{2 P \zeta(2 \delta-1)}{r_{\min }^{2 \delta-1}} n,
$$

for all $\delta>1$. With full CSI at the nodes, the transport capacity is upper bounded by

$$
\frac{4 P A(\delta)}{r_{\min }^{2 \delta-1}} n,
$$

as long as $\delta>3 / 2$. Here $\zeta(\delta):=\sum_{i=1}^{\infty} i^{-\delta}$, a finite number for $\delta>1$, is the Riemann-Zeta function and $A(\delta):=\zeta(\delta)+$ $\frac{1}{2-\delta}\{\zeta(2 \delta-2)-\zeta(\delta)\}$.

Proof: We first consider a regular linear network in which the consecutive nodes are equally spaced on the real line with node $i$ at $r_{\min } i$ for $i \in I=\left\{-\frac{n-1}{2}, \ldots-1,0,1, \ldots, \frac{n-1}{2}\right\} .{ }^{2}$ For such a configuration of nodes, $r_{i j}=r_{\min }|i-j|$ for $i, j \in I$. We evaluate the expression (5) by observing that choosing $j=0$ yields the largest inner sum (over all $i$ ):

$$
\begin{aligned}
C_{T} & \leq \sum_{i, j \neq i} P_{i} \frac{r_{\min }|i-j|}{\left(1+r_{\min }|i-j|\right)^{2 \delta}}, \\
& <\sum_{i, j \neq i} \frac{P_{i}}{\left(1+r_{\min }|i-j|\right)^{2 \delta-1}}, \\
& \leq 2 P n \sum_{i=1}^{(n-1) / 2} \frac{1}{\left(1+r_{\min } i\right)^{2 \delta-1}}, \\
& <\frac{2 P \zeta(2 \delta-1)}{r_{\min }^{2 \delta-1}} n .
\end{aligned}
$$

${ }^{2}$ Since we are deriving an upper bound we can always, if necessary, add another node to make $n$ odd. 
Now suppose the nodes are arbitrarily positioned on a line with $r_{\text {min }}$ being the smallest distance between any two nodes. Then we can move the nodes in by shrinking the distance between any two consecutive ones down to $r_{\min }$ so that for $i \neq j, r_{i j} \geq r_{\min }|i-j|$. Consequently, the upper bound to transport capacity is largest for the regular linear network and we have shown the first part of the corollary.

Let us turn to the full CSI model. Again it suffices to consider regular linear networks. Since now the nodes have full CSI we must evaluate the expression (6). We again observe that setting $j=0$ yields an inner sum (over all other nodes $i, k$ ) larger than for any other node $j$. Thus,

$$
\begin{aligned}
C_{T} & \leq \sum_{\substack{i, k \\
j \neq i, k}} \sqrt{P_{i} P_{k}} \frac{r_{\min } \min (|i-j|,|k-j|)}{\left(1+r_{\min }|i-j|\right)^{\delta}\left(1+r_{\min }|k-j|\right)^{\delta}}, \\
& \leq 2 P n \sum_{i, k=1}^{(n-1) / 2} \frac{r_{\min } \min (i, k)}{\left(1+r_{\min } i\right)^{\delta}\left(1+r_{\min } k\right)^{\delta}}, \\
& \leq 4 P n \sum_{i=1}^{(n-1) / 2} \sum_{k \geq i} \frac{1}{\left(1+r_{\min } i\right)^{\delta-1}\left(1+r_{\min } k\right)^{\delta}}, \\
& <\frac{4 P n}{r_{\min }^{2 \delta-1}} \sum_{k=1}^{n} \sum_{i=1}^{k} \frac{1}{i^{\delta-1} k^{\delta}} .
\end{aligned}
$$

We are interested in $\delta>1$, the decay rate even in free space, where we have the lower Riemann sum

$$
\begin{aligned}
\sum_{i=1}^{k} i^{1-\delta} & <1+\int_{1}^{k} x^{1-\delta} d x \\
& <1+\frac{1}{2-\delta}\left(k^{2-\delta}-1\right)
\end{aligned}
$$

Using (15) to upper bound the inner sum in (14), we get

$$
\sum_{k=1}^{n} \sum_{i=1}^{k} i^{1-\delta} k^{-\delta} \leq \zeta(\delta)+\frac{1}{2-\delta}\{\zeta(2 \delta-2)-\zeta(\delta)\} .
$$

The above constant is well-defined for all $\delta>3 / 2+\epsilon$ : It is upper bounded by $\zeta(\delta)+2 \zeta(1+2 \epsilon)$ and decreases monotonically to zero as $\delta$ grows. Here $\epsilon>0$ is arbitrary. Denoting the constant in $(16)$ by $A(\delta)$ we obtain the statement of our claim in (13).

The following corollary establishes a similar result for planar networks.

Corollary 3.2: Suppose that the nodes lie on a plane at a distance of at least $r_{\min }>0$ from each other. With no CSI at the nodes, the transport capacity is upper bounded by

$$
\frac{(2 \pi+12) P \zeta(2 \delta-2)}{r_{\min }^{2 \delta-1}} n,
$$

for $\delta>3 / 2$. With full CSI at the nodes, the transport capacity is upper bounded by

$$
\frac{2(2 \pi+12)^{2} P A(\delta-1)}{r_{\min }^{2 \delta-1}} n,
$$

for $\delta>5 / 2$.

Proof: Consider an arbitrary planar network with minimum distance $r_{\min }>0$ and let us begin with the no CSI model.
To upper bound the transport capacity we need to evaluate the expression in (5).

$$
\begin{aligned}
C_{T} & \leq \sum_{i, j \neq i} P_{i} \frac{r_{i j}}{\left(1+r_{i j}\right)^{2 \delta}} \\
& <\sum_{i, j \neq i} \frac{P_{i}}{r_{i j}^{2 \delta-1}} .
\end{aligned}
$$

We pick one of the nodes, say $i_{0}$, and wish to evaluate the inner sum (over all $j \neq i_{0}$ ) in (5). The value of this quantity will depend upon the actual geographic configuration of the nodes. However, we can find an upper bound to it by counting the greatest possible number of nodes at any given distance from $i_{0}$. Observe that at a distance of $j r_{\min }$ from $i_{0}$ there are at most $\lfloor 2 \pi j\rfloor$ nodes. Suppose that we move the nodes lying in the annulus formed by circles of radius $j r_{\min }$ and $(j+1) r_{\min }$ to a distance of $j r_{\min }$ from node $i_{0}$. Since there are at most

$$
\frac{\pi\left(r_{\min }(j+1)\right)^{2}-\pi\left(r_{\min } j\right)^{2}}{\pi\left(\frac{r_{\min }}{2}\right)^{2}}=4(2 j+1)
$$

such nodes, the overall number of nodes at distance $j r_{\min }$ from node $i_{0}$ cannot be larger than $\lfloor(2 \pi+8) j+4\rfloor$. Note that moving nodes closer to $i_{0}$ only further upper bounds the quantity $\sum_{i_{0}, j \neq i_{0}} \frac{1}{r_{i_{0} j}^{2 \delta-1}}$. Continuing from (18), our upper bound on transport capacity is

$$
\begin{aligned}
C_{T} & <\frac{P n}{r_{\min }^{2 \delta-1}} \sum_{j=1}^{\sqrt{n} / 2} \frac{\lfloor(2 \pi+8) j+4\rfloor}{j^{2 \delta-1}}, \\
& <\frac{P n}{r_{\min }^{2 \delta-1}} \sum_{j=1}^{\sqrt{n} / 2} \frac{(2 \pi+12) j}{j^{2 \delta-1}} \\
& <\frac{(2 \pi+12) P \zeta(2 \delta-2) n}{r_{\min }^{2 \delta-1}}
\end{aligned}
$$

This proves the first part of the corollary.

Now consider the full CSI model. This time we evaluate the expression (6) to bound the transport capacity. We use the same counting arguments as those used in the proof of the first statement of this corollary to get

$$
\begin{aligned}
C_{T} & \leq \sum_{i, k, j \neq i, k} \sqrt{P_{i} P_{k}} \frac{\min \left(r_{i j}, r_{k j}\right)}{\left(1+r_{i j}\right)^{\delta}\left(1+r_{k j}\right)^{\delta}}, \\
& \leq \frac{(2 \pi+12)^{2} P n}{r_{\min }^{2 \delta-1}} \sum_{i, k=1}^{\sqrt{n} / 2} \frac{i k \min (i, k)}{i^{\delta} k^{\delta}}, \\
& \leq \frac{2(2 \pi+12)^{2} P n}{r_{\min }^{2 \delta-1}} \sum_{i=1}^{\sqrt{n} / 2} \sum_{k \geq i} \frac{1}{i^{\delta-2} k^{\delta-1}} \\
& <\frac{2(2 \pi+12)^{2} P n}{r_{\min }^{2 \delta-1}} \sum_{k=1}^{n} \sum_{i=1}^{k} \frac{1}{i^{\delta-2} k^{\delta-1}} .
\end{aligned}
$$

Since the only difference here from (14) is that $\delta$ is reduced by 1 , we have shown the claim in (17).

\section{SCAling LAWS FOR RANDOM NETWORKS}

In the previous section we considered arbitrary linear and planar network configurations under the assumption that no 
two nodes can be closer than a distance of $r_{\min }>0$. To test the robustness and generality of our approach, we introduce random perturbations in the locations of the nodes. As a first step to tackling this problem, we observe the following simple result, a proof of which can be found in Section 3 of [6].

Given a random process $\left\{\mathcal{X}_{n}\right\}_{n=1}^{\infty}$ and functions $f$ and $g$, we will use the shorthand notation $f\left(\mathcal{X}_{n}\right) \lesssim g(n)$ to mean the following:

$$
\mathbb{P}\left(f\left(\mathcal{X}_{n}\right) \leq g(n) \text { for all but finitely many } n\right)=1 .
$$

Lemma 4.1: Suppose there are $n \geq 1$ urns and we are given $n$ indistinguishable balls to distribute among them. Further, suppose that we are equally likely to place any of the balls in any of the urns. Let $\mathcal{B}_{n}=\left\{i_{1}, i_{2}, \ldots, i_{n}\right\}$ represent a realization of the locations of $n$ balls in $n$ urns, i.e., let $i_{k}$ be the number of balls that fall in urn $k$. Thus $\left\{\mathcal{B}_{n}\right\}_{n=1}^{\infty}$ is a random process, indexed by $n$, of realizations of ball locations. Then, we have that $\max \mathcal{B}_{n} \lesssim \log n$.

We now proceed to apply this result to linear and planar random networks. Let $\left\{\mathcal{N}_{n}\right\}_{n=1}^{\infty}$ denote the random process of realizations of network configurations, indexed by the number of radios $n$. We will write $C_{T}\left(\mathcal{N}_{n}\right)$ to emphasize the fact that the transport capacity is a function of this random process.

Theorem 4.2: Suppose that $\mathcal{N}_{n}$ is a collection of $n$ nodes whose locations are independently and uniformly distributed on a straight line of length $n$. With no CSI at the nodes, we have that

$$
C_{T}\left(\mathcal{N}_{n}\right) \lesssim 4 P \zeta(2 \delta-1) n \log n,
$$

for all $\delta>1$. With full CSI at the nodes, we have that

$$
C_{T}\left(\mathcal{N}_{n}\right) \lesssim 16 P A(\delta) n(\log n)^{2},
$$

as long as $\delta>3 / 2$.

Proof: Suppose that the $n$ nodes in the network $\mathcal{N}_{n}$ have no CSI and that their locations are uniformly and independently distributed on the line segment $[0, n]$. We will use letters $x$ and $y$ to denote the nodes (and, interchangeably, their locations) in $\mathcal{N}_{n}$, and $|A|$ to denote the cardinality of set $A$. Identifying the urns in Lemma 4.1 with the intervals $[i, i+1]$, we get that $\left|\mathcal{N}_{n} \cap[i, i+1]\right| \lesssim \log n$ for each $i \in I_{n} \triangleq\{0,1, \ldots, n\}$.

Suppose that, given a fixed $j \in I_{n}$, we move the nodes lying in $[i, i+1]$ to the point $i$ if $j \leq i$ or to the point $i+1$ if $j>i$. At the remaining points in $I_{n}$ we add $\log n$ nodes, since this can also only further increase transport capacity. The result is a regular linear network with at most (in the sense of "¿”) $2 \log n$ nodes at distance $r_{i j}$ from $j$, for each $i \in I_{n}$. We now use this construction of regular networks to upper bound expression (5) for the random network:

$$
\begin{aligned}
C_{T}\left(\mathcal{N}_{n}\right) & <\sum_{x, y \in \mathcal{N}_{n}: x \neq y} \frac{P_{x}}{\left(1+r_{x y}\right)^{2 \delta-1}}, \\
& \lesssim \sum_{j \in I_{n}} \sum_{i \in I_{n}} \frac{2 P \log n}{\left(1+r_{i j}\right)^{2 \delta-1}}, \\
& <4 P n \log n \sum_{k=0} \frac{1}{(1+k)^{2 \delta-1}}, \\
& <4 P \zeta(2 \delta-1) n \log n .
\end{aligned}
$$

Here, as before, we use $P$ to denote $\max _{x \in \mathcal{N}_{n}} P_{x}$. The inequality (20) was obtained by observing that choosing $j=$ $n / 2$ yields the largest inner sum (over $i$ ). Note that the bound is valid only for $\delta>1$. Thus the first part of the theorem is proved.

Now suppose the nodes have full CSI. A procedure identical to the one described above is used to generate, for each $j \in$ $I_{n}$, a regular linear network with at most $2 \log n$ nodes at each distance $r_{i j}$ from $j$. We then proceed to upper bound expression (6):

$$
\begin{aligned}
C_{T}\left(\mathcal{N}_{n}\right) & <\sum_{x, y, z \in \mathcal{N}_{n}: z \neq x, y} \sqrt{P_{x} P_{y}} \frac{1+\min \left(r_{x z}, r_{y z}\right)}{\left(1+r_{x z}\right)^{\delta}\left(1+r_{y z}\right)^{\delta}}, \\
& \lesssim \sum_{j \in I_{n}} \sum_{i, k \in I_{n}} 4 P(\log n)^{2} \frac{1+\min \left(r_{i j}, r_{k j}\right)}{\left(1+r_{i j}\right)^{\delta}\left(1+r_{k j}\right)^{\delta}}, \\
& \leq 8 P n(\log n)^{2} \sum_{l, m=0}^{n / 2} \frac{1+\min (l, m)}{(1+l)^{\delta}(1+m)^{\delta}}, \\
& <16 P n(\log n)^{2} \sum_{m=1}^{n} \sum_{l=1}^{m} \frac{1}{l^{\delta-1} m^{\delta}} .
\end{aligned}
$$

Inequality (21) was obtained using the observation that choosing $j=n / 2$ yields the largest inner sum (over all $i, k$ ). With the notation from (16) we get that, for $\delta>3 / 2$,

$$
C_{T}\left(\mathcal{N}_{n}\right) \lesssim 16 P A(\delta) n(\log n)^{2},
$$

which proves the second part of the theorem.

Theorem 4.3: Suppose that $\mathcal{N}_{n}$ is a collection of $n$ nodes whose locations are independently and uniformly distributed on a square of area $n$. With no CSI at the nodes, we have that

$$
C_{T}\left(\mathcal{N}_{n}\right) \lesssim 4 P(2 \zeta(2 \delta-2)+\zeta(2 \delta-1)) n \log n
$$

as long as $\delta>3 / 2$. With full CSI at the nodes, we have that

$C_{T}\left(\mathcal{N}_{n}\right) \lesssim 32\{4 A(\delta-1)+4 B(\delta)+A(\delta)\} P n(\log n)^{2}$, as long as $\delta>5 / 2$. Here

$$
B(\delta):=\zeta(\delta-1)+\frac{1}{\delta-2}(\zeta(\delta-1)-\zeta(2 \delta-3))
$$

is a finite number for $\delta>5 / 2$.

Proof: Assume that the nodes have no CSI. Suppose that we partition the square (of area $n$ ) in which the network $\mathcal{N}_{n}$ lies, into $n$ square-lets $s_{1}, s_{2}, \ldots, s_{n}$, each of unit area. This forms a regular grid: $\mathbb{S}_{n}:=\{(i, j): i, j \in\{0,1, \ldots, \sqrt{n}\}\} \subset \mathbb{Z}^{2}$. Identifying the urns in Lemma 4.1 with the square-lets $s_{i}$, we get that $\left|\mathcal{N}_{n} \cap s_{i}\right| \lesssim \log n$ for each $i \in I_{n}$. Now, pick any vertex $\mathbf{v} \in \mathbb{S}_{n}$. Suppose that we move the nodes that fall in each square-let of $\mathbb{S}_{n}$ onto the square-let vertex that is the closest, in the Euclidean sense, to $\mathbf{v}$. At the remaining "empty" vertices in $\mathbb{S}_{n}$ we add $\log n$ nodes, since this can also only further increase transport capacity. This will result in a regular planar network but with multiple nodes at each vertex. In particular, there will be at most (in the sense of " $\lesssim$ ") $2 \log n$ nodes at each vertex in some subset $\widetilde{\mathbb{S}}_{n} \subset \mathbb{S}_{n} \backslash\{\mathbf{v}\}$, at most $\log n$ nodes at each vertex in the complement of $\widetilde{\mathbb{S}}_{n}$ in $\mathbb{S}_{n}$, and at most $4 \log n$ nodes at $\mathbf{v}$ (see Figure 2). In other 


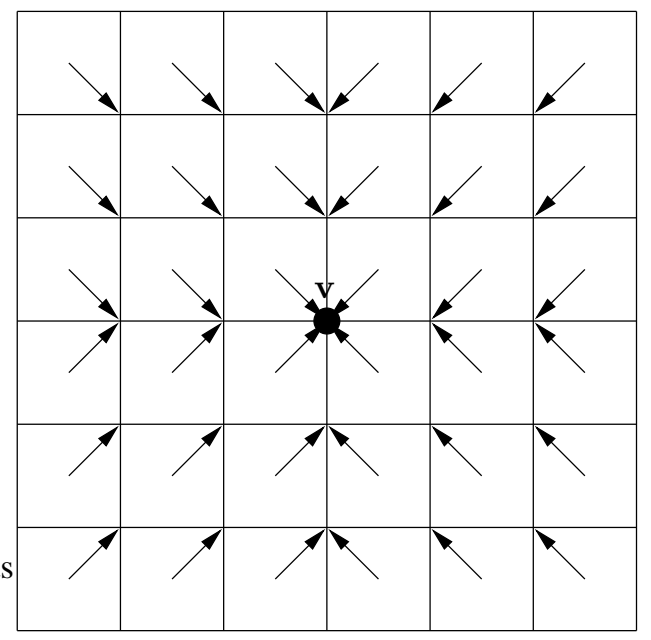

Fig. 2. The grid $\mathbb{S}_{36}$ for a random network on a plane. As indicated by the arrows, the nodes in each square-let are moved to the square-let vertex closest to vertex $\mathbf{v}$.

words, the largest possible number of nodes at any $\mathbf{u} \in \mathbb{S}_{n}$ is $\left(1+\mathbf{1}_{\left\{\mathbf{u} \in \widetilde{\mathbb{S}}_{n}\right\}}+3 \cdot \mathbf{1}_{\{\mathbf{u}=\mathbf{v}\}}\right) \log n=: f_{\mathbf{v}}(\mathbf{u})$.

Now, let $\mathbf{v}$ be the central node. Then we see that on the square of perimeter $8 k$ centered at $\mathbf{v}$ there are $8 k$ vertices, for $k \in\{1,2, \ldots, \sqrt{n} / 2\}$. By inspecting Figure 2 we see that four of these vertices will have at most $2 \log n$ nodes and the rest, $8 k-4$ vertices, will have at most $\log n$ nodes. Suppose that we move all of the nodes at these vertices onto the circle of radius $k$ centered at $\mathbf{v}$. Then we will get a total of at most $(8 k+4) \log n$ nodes at each distance $k \in\{0,1, \ldots, \sqrt{n} / 2\}$ from $\mathbf{v}$. We then have, starting from expression (5), the following sequence of upper bounds:

$$
\begin{aligned}
C_{T}\left(\mathcal{N}_{n}\right) & \leq \sum_{x, y \in \mathcal{N}_{n}: x \neq y} \frac{P_{x}}{\left(1+r_{x y}\right)^{2 \delta-1}}, \\
& \lesssim \sum_{\mathbf{v} \in \mathbb{S}_{n}} \sum_{\mathbf{u} \in \mathbb{S}_{n}} P \frac{f_{\mathbf{v}}(\mathbf{u}) \log n}{(1+\|\mathbf{v}-\mathbf{u}\|)^{2 \delta-1}}, \\
& \leq \operatorname{Pn} \log n \sum_{k=0}^{\sqrt{n} / 2} \frac{(8 k+4)}{(1+k)^{2 \delta-1}}, \\
& <4 \operatorname{Pn} \log n\left\{2 \sum_{k=1}^{\frac{\sqrt{n}}{2}+1} \frac{1}{k^{2 \delta-2}}+\sum_{k=1}^{\frac{\sqrt{n}}{2}+1} \frac{1}{k^{2 \delta-1}}\right\} .
\end{aligned}
$$

We thus arrive at the claimed upper bound, for $\delta>3 / 2$,

$$
C_{T}\left(\mathcal{N}_{n}\right) \lesssim 4 P(2 \zeta(2 \delta-2)+\zeta(2 \delta-1)) n \log n
$$

Now suppose that the network nodes have full CSI. A procedure identical to the one described above is used to generate, for each $\mathbf{v} \in \mathbb{S}_{n}$, a regular planar network with at most (in the sense of "¿”) $f_{\mathbf{v}}(\mathbf{u}) \log n$ nodes at each vertex $\mathbf{u} \in \mathbb{S}_{n}$. We then have

$$
\begin{aligned}
& C_{T}\left(\mathcal{N}_{n}\right) \leq \sum_{\substack{x, y, z \in \mathcal{N}_{n}: \\
z \neq x, y}} \sqrt{P_{x} P_{y}} \frac{1+\min \left(r_{x z}, r_{y z}\right)}{\left(1+r_{x z}\right)^{\delta}\left(1+r_{y z}\right)^{\delta}}, \\
& \lesssim \sum_{\mathbf{v}, \mathbf{u}, \mathbf{t} \in \mathbb{S}_{n}} P(\log (n))^{2} f_{\mathbf{v}}(\mathbf{u}) f_{\mathbf{v}}(\mathbf{t}) \text {. } \\
& \frac{(1+\min (\|\mathbf{u}-\mathbf{v}\|,\|\mathbf{t}-\mathbf{v}\|))}{(1+\|\mathbf{u}-\mathbf{v}\|)^{\delta}(1+\|\mathbf{t}-\mathbf{v}\|)^{\delta}}, \\
& \leq \operatorname{Pn}(\log n)^{2} \text {. } \\
& \sum_{j, k=0}^{\sqrt{n} / 2} \frac{(8 j+4)(8 k+4)(1+\min (j, k))}{(1+j)^{\delta}(1+k)^{\delta}}, \\
& <32\{4 A(\delta-1)+4 B(\delta)+A(\delta)\} P n(\log n)^{2} .
\end{aligned}
$$

Here the last step follows from observing the similarity to (21) with $\delta$ replaced by $\delta-1$. Thus the condition on $\delta$ for the upper bound to hold is now $\delta>5 / 2$.

\section{Scaling Laws for Networks with a Total Power CONSTRAINT}

We have considered networks in which the nodes are individually power limited and have observed essentially $O(n)$ scaling of upper bounds to transport capacity of minimum distance and random networks. In this section, we would like to study the sensitivity of this scaling law to individual power constraints of the nodes. In particular, we allow arbitrary distribution of power to the radios in the network (constraining the total transmit power to grow linearly in the number of radios). We will see that with this extra degree of flexibility, approximately $n^{\frac{1}{2 \delta-1}}$ parallel channels can be created in the cut-set bound. As before, we begin with linear networks then moving on to planar networks.

Theorem 5.1: Suppose the nodes lie on a line at a distance of at least $r_{\min }>0$ from each other. Further, the signal decay parameter $\delta>1$. With no CSI at the nodes, the transport capacity is upper bounded by

$$
\pi\left(\frac{P}{r_{\min }^{2 \delta}}+\frac{2 \delta-1}{2 \delta}\right) n^{1+\frac{1}{2 \delta}} \log n .
$$

With full CSI at the nodes, the transport capacity is upper bounded by

$\pi n\left(\log \left(1+\frac{P n}{r_{\min }^{2 \delta}}\right) n^{\frac{1}{2 \delta-1}}+\frac{P}{r_{\min }^{2 \delta}} n^{\frac{1}{2 \delta-1}}+(2 \delta-1)\right)$.

Proof: We begin with no CSI at the nodes. Suppose that the nodes are allowed to distribute a total power of $n P$ among each other. Then, by partitioning the network with a uniformly random line $L$, we will observe a total power of, say, $n P^{+}$ in $\mathcal{C}^{+}$and $n P^{-}=n\left(P-P^{+}\right)$in $\mathcal{C}^{-}$. Examining (10), we observe that transport capacity can also be expressed as the supremum (over all achievable rates) of

$$
\pi d_{n} \mathbb{E}_{L}\left[\sum_{i \in \mathcal{C}^{+}, j \in \mathcal{C}^{-}} R_{i j}+\sum_{i \in \mathcal{C}^{-}, j \in \mathcal{C}^{+}} R_{i j}\right],
$$

where $\mathbb{E}_{L}$ denotes the expectation operator with respect to the distribution of the line $L$. Now, applying the MIMO channel 
bound with a total power constraint of $n P^{+}$to the first sumrate in the above we expression we obtain

$$
\sum_{i \in \mathcal{C}^{+}, j \in \mathcal{C}^{-}} R_{i j}<\max _{\substack{\mathbf{K}_{x} \succeq 0: \\ \operatorname{Tr}\left(\mathbf{K}_{x}\right) \leq n P^{+}}} \mathbb{E}\left[\log \operatorname{det}\left(\mathbf{I}+\mathbf{H K}_{x} \mathbf{H}^{\dagger}\right)\right],
$$

where $\mathbf{H} \in \mathbb{C}^{n_{\mathcal{C}^{-}} \times n_{\mathcal{C}^{+}}}$. A similar expression is true for the second term but with $\mathbf{H}$ replaced by $\mathbf{H}^{\dagger}$ and $P^{+}$replaced by $P^{-}$. We first claim that the optimal covariance matrix of this maximization problem is again diagonal, allowing the reduction of the constraint set to all p.s.d. diagonal matrices $\mathbf{P}=\operatorname{diag}\left(p_{1}, p_{2}, \ldots, p_{n_{\mathcal{C}^{+}}}\right)$for which the trace constraint is satisfied (see (39) in the appendix for the justification). We also note that the nonzero eigenvalues of $\mathbf{H P H}^{\dagger}$ are equal to the nonzero eigenvalues of $\mathbf{P H}^{\dagger} \mathbf{H}$ so that

$$
\sum_{i \in \mathcal{C}^{+}, j \in \mathcal{C}^{-}} R_{i j}<\max _{\substack{\mathbf{P} \succeq 0: \\ \operatorname{Tr}(\mathbf{P}) \leq n P^{+}}} \mathbb{E}\left[\log \operatorname{det}\left(\mathbf{I}+\mathbf{P} \mathbf{H}^{\dagger} \mathbf{H}\right)\right] .
$$

Applying the Hadamard bound and Jensen's inequality and then adding the two sum-rate terms we get that

$$
\begin{aligned}
& \frac{C_{T}}{\pi d_{n}} \leq \\
& \mathbb{E}_{L}\left[\max _{\mathbf{p} \in S\left(n_{\left.\mathcal{C}^{+}, P^{+}\right)}\right.} \sum_{i \in \mathcal{C}^{+}} \log \left(1+p_{i} \sum_{j \in \mathcal{C}^{-}} \frac{1}{\left(1+r_{i j}\right)^{2 \delta}}\right)\right. \\
& \left.+\max _{\tilde{\mathbf{p}} \in S\left(n_{\mathcal{C}^{-}}, P^{-}\right)} \sum_{i \in \mathcal{C}^{-}} \log \left(1+\tilde{p}_{i} \sum_{j \in \mathcal{C}^{+}} \frac{1}{\left(1+r_{i j}\right)^{2 \delta}}\right)\right]
\end{aligned}
$$

where we have used $\widetilde{\mathbf{p}}$ to denote the powers of the nodes in $\mathcal{C}^{-}$, and where we have defined the set

$$
S(k, T):=\left\{\mathbf{p}=\left(p_{1}, p_{2}, \ldots, p_{k}\right) \in \mathbb{R}_{+}^{k}: \sum p_{i} \leq n T\right\} .
$$

Now, allowing $j \in\{1,2, \ldots, n\}$, and collecting the two terms we obtain a further upper bound:

$$
\begin{aligned}
\frac{C_{T}}{\pi d_{n}} & \leq \max _{\mathbf{p} \in S(n, P)} \sum_{i=1}^{n} \log \left(1+p_{i} \sum_{j=1}^{n} \frac{1}{\left(1+r_{i j}\right)^{2 \delta}}\right) \\
& =: M_{n} .
\end{aligned}
$$

Since the function that is being maximized is concave and is defined on a convex compact set, the maximum value is also given by the dual problem:

$$
M_{n}=\min _{\lambda \geq 0} \max _{\mathbf{p} \in \mathbb{R}_{+}^{n}} L(\lambda, \mathbf{p}) .
$$

Here $L(\lambda, \mathbf{p})$ is the Lagrangian of the primal problem and is given by

$$
L(\lambda, \mathbf{p})=\sum_{i=1}^{n} \log \left(1+p_{i} V_{i}\right)-\lambda\left(\sum_{i=1}^{n} p_{i}-n P\right) .
$$

We have used $V_{i}$ to denote $\sum_{j=1}^{n} \frac{1}{\left(1+r_{i j}\right)^{2 \delta}}$. Classical waterpouring of $\left\{p_{i}\right\}$ over $\left\{V_{i}\right\}$ tells us that the maximum of $L(\lambda, \mathbf{p})$ over $\mathbf{p} \in \mathbb{R}_{+}^{n}$ is given by

$$
\sum_{i=1}^{n} \log ^{+}\left(\frac{V_{i}}{\lambda}\right)-\lambda \sum_{i=1}^{n}\left(\frac{1}{\lambda}-\frac{1}{V_{i}}\right)^{+}+\lambda n P .
$$

Here we have defined $x^{+}=\max (x, 0)$ and the function $\log ^{+}(x)=\log (x)$ if $x \geq 1$ and zero otherwise.

As in Section III it suffices to consider a regular linear network with $n$ nodes spaced by a distance of exactly $r_{\min }>$ 0 . Further, we only focus on $r_{\min } \leq 1$ because, according to (24), the smaller the node separation, the greater the upper bound on transport capacity. Note that for a linear regular network at $r_{\min }$ we have that $r_{i j}=r_{\min }(i+j-1)$. Thus by the upper Riemann sum,

$$
\begin{aligned}
V_{i} & =\sum_{j=1}^{n} \frac{1}{\left(1+r_{\min }(i+j-1)\right)^{2 \delta}}, \\
& <\int_{0}^{n} \frac{d x}{\left(r_{\min }(i+x)+1-r_{\min }\right)^{2 \delta}}, \\
& <\frac{1 / r_{\min }}{\left(r_{\min } i+1-r_{\min }\right)^{2 \delta-1}}, \\
& \leq \frac{1}{r_{\min }^{2 \delta}\left(i+\frac{1}{r_{\min }}-1\right)^{2 \delta-1}}, \\
& \frac{1}{r_{\min }^{2 \delta} i^{2 \delta-1}} .
\end{aligned}
$$

We choose $\lambda=r_{\min }^{-2 \delta} n^{\frac{1-2 \delta}{2 \delta}}$ and use (28) to obtain

$$
\begin{aligned}
M_{n} & <\sum_{i=1}^{\left\lfloor n^{\frac{1}{2 \delta}}\right\rfloor} \log \left(\frac{r_{\min }^{2 \delta} n^{\frac{2 \delta-1}{2 \delta}}}{r_{\min }^{2 \delta} i^{2 \delta-1}}\right)+\frac{P n^{\frac{1}{2 \delta}}}{r_{\min }^{2 \delta}} \\
& <\frac{2 \delta-1}{2 \delta} n^{\frac{1}{2 \delta}} \log n+\frac{P}{r_{\min }^{2 \delta}} n^{\frac{1}{2 \delta}} \\
& =\left(\frac{P}{r_{\min }^{2 \delta}}+\frac{2 \delta-1}{2 \delta}\right) n^{\frac{1}{2 \delta}} \log n .
\end{aligned}
$$

Substituting this calculation into the upper bound in (25), we obtain the statement of the theorem.

Now consider the full CSI model. The usual bound on the sum-rate of communication from $\mathcal{C}^{+}$to $\mathcal{C}^{-}$is

$$
\begin{aligned}
\sum_{\substack{i \in \mathcal{C}^{+} \\
j \in \mathcal{C}^{-}}} R_{i j} & <\mathbb{E}\left[\max _{\substack{\mathbf{K}_{x} \succeq 0: \\
\operatorname{Tr}\left(\mathbf{K}_{x}\right) \leq n P^{+}}} \log \operatorname{det}\left(\mathbf{I}+\mathbf{H} \mathbf{K}_{x} \mathbf{H}^{\dagger}\right)\right], \\
& \leq \mathbb{E}\left[\sum_{j \in \mathcal{C}^{-}} \max _{\substack{\operatorname{Tr}\left(\mathbf{K}_{x} \succeq 0: \leq n P^{+}\\
\\
\right.}} \log \left(1+\mathbf{h}_{j} \mathbf{K}_{x} \mathbf{h}_{j}^{\dagger}\right)\right] .
\end{aligned}
$$

Here we have, again, used $\mathbf{h}_{j}$ to denote the $j$-th row of $\mathbf{H}$. Now note that, for any given row vector $\mathbf{x} \in \mathbb{C}^{n}$, the maximum of $\mathbf{x} \mathbf{K}_{x} \mathbf{x}^{\dagger}$ over all p.s.d. matrices $\mathbf{K}_{x}$ with unit trace constraint is achieved by $\mathbf{K}_{x}=\frac{\mathbf{x}^{\dagger} \mathbf{x}}{\|\mathbf{x}\|^{2}}$. Therefore,

$$
\max _{\substack{\mathbf{K}_{x} \succeq 0: \\ \operatorname{Tr}\left(\mathbf{K}_{x}\right) \leq n P^{+}}} \mathbf{h}_{j} \mathbf{K}_{x} \mathbf{h}_{j}^{\dagger}=n P^{+}\left\|\mathbf{h}_{j}\right\|^{2} .
$$

Now, using Jensen's inequality to further upper bound the sumrates in both directions across the cut and then averaging the 
result over the distribution of the cut, we arrive at

$$
\begin{aligned}
\frac{C_{T}}{\pi d_{n}} \leq \mathbb{E}_{L} & {\left[\sum_{j \in \mathcal{C}^{-}} \log \left(1+n P^{+} \sum_{i \in \mathcal{C}^{+}} \frac{1}{\left(c+r_{i j}\right)^{2 \delta}}\right)\right.} \\
& \left.+\sum_{j \in \mathcal{C}^{+}} \log \left(1+n P^{-} \sum_{i \in \mathcal{C}^{-}} \frac{1}{\left(c+r_{i j}\right)^{2 \delta}}\right)\right] .
\end{aligned}
$$

Just as in the proof of the first part of the theorem, we can further upper bound transport capacity by letting $i \in$ $\{1,2, \ldots, n\}$ to get

$$
\frac{C_{T}}{\pi d_{n}} \leq \sum_{i=1}^{n} \log \left(1+n P V_{i}\right)
$$

We substitute the approximation (28) into the above expression, and bound the resulting sum with the following integral:

$$
\begin{aligned}
\frac{C_{T}}{\pi d_{n}}< & \int_{0}^{n} \log \left(1+\frac{P}{r_{\min }^{2 \delta}} \frac{n}{x^{2 \delta-1}}\right) d x \\
= & \int_{0}^{1} \log \left(1+\frac{P}{r_{\min }^{2 \delta}} \frac{n}{x^{2 \delta-1}}\right) d x \\
& +\int_{1}^{n^{\frac{1}{2 \delta-1}}} \log \left(1+\frac{P}{r_{\min }^{2 \delta}} \frac{n}{x^{2 \delta-1}}\right) d x \\
& +\int_{n^{\frac{1}{2 \delta-1}}}^{n} \log \left(1+\frac{P}{r_{\min }^{2 \delta}} \frac{n}{x^{2 \delta-1}}\right) d x \\
\leq \quad & \log \left(1+\frac{P n}{r_{\min }^{2 \delta}}\right)+(2 \delta-1) \int_{0}^{1} \log \left(\frac{1}{x}\right) d x \\
& +\log \left(1+\frac{P n}{r_{\min }^{2 \delta}}\right)\left[n^{\frac{1}{2 \delta-1}}-1\right] \\
& +\int_{n^{\frac{1}{2 \delta-1}}}^{n} \frac{P n}{r_{\min }^{2 \delta} x^{2 \delta-1}} d x, \\
<\quad & (2 \delta-1)+\log \left(1+\frac{P n}{r_{\min }^{2 \delta}}\right) n^{\frac{1}{2 \delta-1}}+\frac{P}{r_{\min }^{2 \delta}} n^{\frac{1}{2 \delta-1}}
\end{aligned}
$$

Observing that $d_{n}=n$ for linear networks, we obtain the statement of the theorem.

Theorem 5.2: Suppose the nodes lie on a plane at a distance of at least $r_{\min }>0$ from each other. With no CSI at the nodes, the transport capacity is upper bounded by

$$
\pi P K n^{\frac{1}{2}+\frac{1}{2 \delta-1}}+\pi \frac{2 \delta-2}{2 \delta-1} n^{1+\frac{1}{2 \delta-1}} \log \left(n r_{\min }^{2 \delta-2}\right)
$$

for $\delta>1$ and with $K=\frac{2 \pi+12}{r_{\text {min }}^{2 \delta}}$. With full CSI at the nodes, the transport capacity is upper bounded by

$\pi n\left(\left(n^{\frac{1}{2 \delta-2}}+1\right) \log (1+n P K)+\frac{P K n^{\frac{1}{2 \delta-2}}}{2 \delta-3}+4 \delta-4\right)$,

for $\delta>3 / 2$.

Proof: We begin with the no CSI model first and follow the derivation used in the proof of the first part of Theorem 5.1 to arrive at

$$
\begin{aligned}
& \sum_{i \in \mathcal{C}^{+}, j \in \mathcal{C}^{-}} R_{i j}< \\
& \max _{\mathbf{p} \in S\left(n_{\left.\mathcal{C}^{+}, P^{+}\right)}\right.} \sum_{i \in \mathcal{C}^{+}} \log \left(1+p_{i} \sum_{j \in \mathcal{C}^{-}} \frac{1}{\left(1+r_{i j}\right)^{2 \delta}}\right)
\end{aligned}
$$

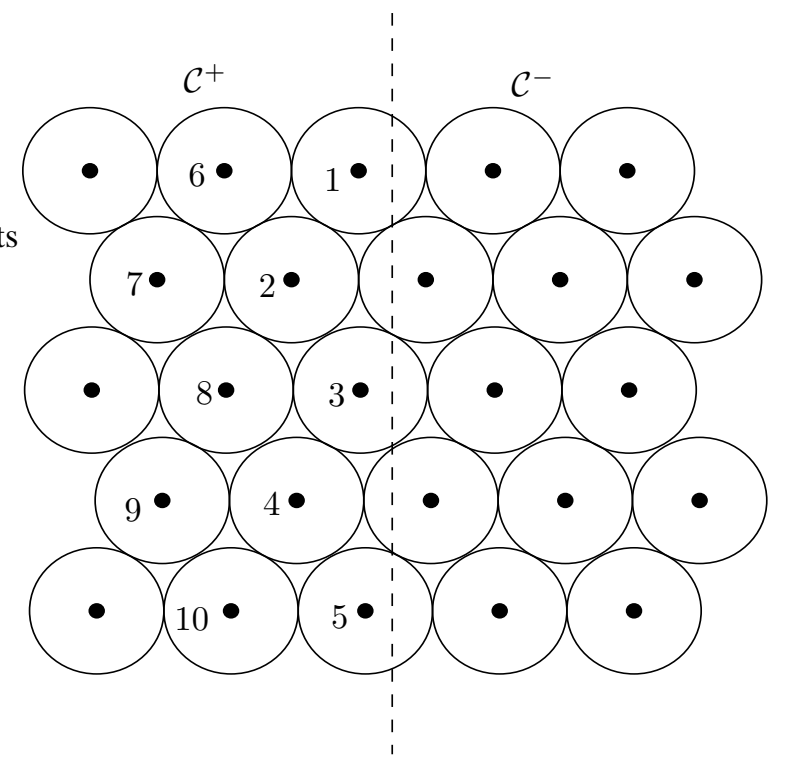

Fig. 3. Hexagonal packing of nodes for $n=25$.

Consider an arbitrary straight line cut of the circle of radius $d_{n}$ containing the $n$ nodes. Consider a new configuration of nodes in $\mathcal{C}^{-}$for which the quantity

$$
V_{i}:=\sum_{j \in \mathcal{C}^{-}} \frac{1}{\left(1+r_{i j}\right)^{2 \delta}}
$$

is maximized for each $i \in \mathcal{C}^{+}$while still honoring the minimum distance property. Then the bound (31) on the sum-rate of communication across this cut will be larger than that of any other node configuration in $\mathcal{C}^{-}$. To maximize our upper bound on overall transport capacity, we would like a single network configuration to have this property for all possible straight-line cuts. Such a minimum-distance network configuration must have the property that each node be surrounded by the greatest possible number of nodes at a distance of $r_{\min }$ from it. To see that this must be so, suppose that there exists a node, call it $i_{0}$, which has one fewer than the maximum number of nodes at distance $r_{\min }$ from it. Suppose further that the node that is missing, call it $j_{0}$, is actually at a distance of $r_{\min }+\epsilon$ from $i_{0}$, for $\epsilon>0$. Then we can choose to partition the network with a straight line that cuts $i_{0}$ and $j_{0}$ and intersects the circle of radius $\frac{r_{\min }}{2}$ around $i_{0}$. Clearly the value of $V_{i_{0}}$ for this cut is less than what it would be if $j_{0}$ was exactly $r_{\text {min }}$ away from $i_{0}$. Thus for a network configuration to be optimal in the sense of maximizing the bound on transport capacity, it must have such a "dense" packing of nodes.

Now, at a distance of $r_{\text {min }}$ from each node there can be at most six other nodes that are also at a distance of $r_{\min }$ from each other. Since this is true for each node, the network configuration that maximizes our bound on transport capacity is the hexagonal packing of nodes in the plane (see Figure 3). We now proceed to upper bound the transport capacity of such a network.

As before, it suffices to consider the center-cut. The enumeration of the nodes in $\mathcal{C}^{+}$is top-to-bottom and according to the pattern indicated in the figure. We claim that $V_{i}$, as a 
function of $i$, changes value only when $\left\lfloor\frac{i-1}{\sqrt{n}}\right\rfloor$ changes value. To see this, observe that for nodes $1 \leq i \leq \sqrt{n}$, the closest node in $\mathcal{C}^{-}$is at a distance of $r_{\text {min }}$. Likewise, for nodes $\sqrt{n}+1 \leq i \leq 2 \sqrt{n}$, the closest receiving node is at a distance of $2 r_{\min }$, and so on. In particular, for $1 \leq i \leq \sqrt{n}$, we have that

$$
V_{i}<\sum_{j=1}^{n} \frac{(2 \pi+12) j}{\left(1+r_{\min } j\right)^{2 \delta}}<\frac{(2 \pi+12)}{r_{\min }^{2 \delta}} \sum_{j=1}^{n} \frac{1}{j^{2 \delta-1}} .
$$

To see this, we use a bounding procedure similar to the one in the proof of Corollary 3.2: We pick any node $i$ in the given set and note that there can be at most $\lfloor(2 \pi+12) j\rfloor$ nodes at distance $r_{\min } j$ from $i$. Note that we can always extend the hexagonal pattern by adding more nodes to ensure that this bound holds for all $1 \leq i \leq \sqrt{n}$. In general, for any $1 \leq i \leq n / 2$, we can write

$$
V_{i}<\frac{(2 \pi+12)}{r_{\min }^{2 \delta}} \sum_{j=1}^{n}\left(\frac{1}{r_{\min }}+j+\left\lfloor\frac{i-1}{\sqrt{n}}\right\rfloor\right)^{1-2 \delta} .
$$

We can then further upper bound the above expression by recognizing it as a Riemann sum:

$$
\begin{aligned}
V_{i} & <\frac{2 \pi+12}{r_{\min }^{2 \delta}} \int_{0}^{n} \frac{d x}{\left(\frac{1}{r_{\min }}+x+\left\lfloor\frac{i-1}{\sqrt{n}}\right\rfloor\right)^{2 \delta-1}}, \\
& <\frac{2 \pi+12}{r_{\min }^{2 \delta}\left(\frac{1}{r_{\min }}+\left\lfloor\frac{i-1}{\sqrt{n}}\right\rfloor\right)^{2 \delta-2}} .
\end{aligned}
$$

Further, we have an upper bound to the transport capacity by choosing $\lambda=\frac{2 \pi+12}{r_{\min }^{2 \delta}} n^{\frac{2-2 \delta}{2 \delta}}$ in the Lagrange dual (c.f. (26)):

$$
\begin{aligned}
\frac{C_{T}}{\pi d_{n}} \leq & \frac{P(2 \pi+12) n^{\frac{1}{2 \delta-1}}}{r_{\min }^{2 \delta}} \\
& +\sum_{i=1}^{n / 2} \log ^{+}\left(n^{\frac{2 \delta-2}{2 \delta-1}}\left\lfloor\frac{1}{r_{\min }}+\frac{i-1}{\sqrt{n}}\right\rfloor^{2-2 \delta}\right)
\end{aligned}
$$

Observe that the number of nonzero terms in the above sum is the same as the number of values of $i$ for which the argument of the $\log ^{+}$function is greater or equal to one. This number is no more than $\left\lfloor\sqrt{n} n^{\frac{1}{2 \delta-1}}\right\rfloor$. Thus we have

$\frac{C_{T}}{\pi d_{n}} \leq \frac{P(2 \pi+12) n^{\frac{1}{2 \delta-1}}}{r_{\min }^{2 \delta}}+\frac{2 \delta-2}{2 \delta-1} \sqrt{n} n^{\frac{1}{2 \delta-1}} \log \left(n r_{\min }^{2 \delta-2}\right)$.

Noting that $d_{n}=\sqrt{n}$ for hexagonally arranged planar networks, we get the desired bound on transport capacity.

Now the nodes have full CSI and we only look at the regular planar network (hexagonally arranged as in Figure 3). We follow the same steps as those used to derive an upper bound for the linear regular network under full CSI in Theorem (5.1), to arrive at the expression (30), which is replicated here for convenience:

$$
\frac{C_{T}}{\pi d_{n}} \leq \sum_{i=1}^{n} \log \left(1+n P V_{i}\right)
$$

Here $V_{i}$ for planar networks is upper bounded as, from (32),

$$
\begin{aligned}
V_{i} & <\frac{2 \pi+12}{r_{\min }^{2 \delta}\left(\frac{1}{r_{\min }}+\left\lfloor\frac{i-1}{\sqrt{n}}\right\rfloor\right)^{2 \delta-2}}, \\
& <\frac{K}{\left(\frac{i}{\sqrt{n}}-1\right)^{2 \delta-2}} .
\end{aligned}
$$

where we have defined the constant $K=\frac{2 \pi+12}{r_{\text {min }}^{2 \delta}}$. If we substitute this upper bound for $V_{i}$ in (34), and note that $d_{n}=\sqrt{n}$ for planar networks, we get

$$
\frac{C_{T}}{\pi \sqrt{n}}<\sum_{i=1}^{n} \log \left(1+n P K\left(\frac{i}{\sqrt{n}}-1\right)^{2-2 \delta}\right) .
$$

We can upper bound this sum with an integral, just as in (28), to obtain

$$
\frac{C_{T}}{\pi \sqrt{n}}<\int_{0}^{n} \log \left(1+n P K\left(\frac{x}{\sqrt{n}}-1\right)^{2-2 \delta}\right) d x .
$$

Performing the change of variable $u=\frac{x}{\sqrt{n}}-1$ and restricting ourselves to $\delta>3 / 2$, we continue to upper bound the integral:

$$
\begin{aligned}
& \frac{C_{T}}{\pi \sqrt{n}}<\int_{-1}^{\sqrt{n}-1} \log \left(1+\frac{n P K}{u^{2 \delta-2}}\right) \sqrt{n} d u, \\
& =\sqrt{n} \int_{-1}^{0} \log \left(1+\frac{n P K}{u^{2 \delta-2}}\right) d u \\
& +\sqrt{n} \int_{0}^{1} \log \left(1+\frac{n P K}{u^{2 \delta-2}}\right) d u \\
& +\sqrt{n} \int_{1}^{n^{\frac{1}{2 \delta-2}}} \log \left(1+\frac{n P K}{u^{2 \delta-2}}\right) d u \\
& +\sqrt{n} \int_{n^{\frac{1}{2 \delta-2}}}^{\sqrt{n}-1} \log \left(1+\frac{n P K}{u^{2 \delta-2}}\right) d u, \\
& =2 \sqrt{n} \int_{0}^{1} \log \left(1+\frac{n P K}{u^{2 \delta-2}}\right) d u \\
& +\sqrt{n} \int_{1}^{n^{\frac{1}{2 \delta-2}}} \log \left(1+\frac{n P K}{u^{2 \delta-2}}\right) d u \\
& +\sqrt{n} \int_{n^{\frac{1}{2 \delta-2}}}^{\sqrt{n}-1} \log \left(1+\frac{n P K}{u^{2 \delta-1}}\right) d u, \\
& <2 \sqrt{n} \log (1+n P K)+2 \sqrt{n}(2 \delta-2) \\
& +\sqrt{n} \log (1+n P K)\left[n^{\frac{1}{2 \delta-2}}-1\right] \\
& +\sqrt{n} \int_{n \frac{1}{2 \delta-2}}^{\sqrt{n}} \frac{n P K}{u^{2 \delta-2}} d u, \\
& <\sqrt{n} \log (1+n P K)+2 \sqrt{n}(2 \delta-2) \\
& +\sqrt{n} \log (1+n P K) n^{\frac{1}{2 \delta-2}}+\sqrt{n} \frac{P K n^{\frac{1}{2 \delta-2}}}{2 \delta-3} .
\end{aligned}
$$

\section{DISCUSSION}

We have applied our simple upper bounds to transport capacity to calculate an upper bound to scaling laws for certain class of network topologies. While this bound matched the scaling law with a simple multihop communication scheme, care must be exercised in interpreting this result. In particular, 
the preconstants for the lower bound and the upper bound to the transport capacity are different and have to be taken into consideration when architecting the communication strategy.

A key aspect to our study of scaling laws has been the consideration of minimum distance networks (or random networks over a large area). The scenario where there is a crowding of nodes in a fixed area is interesting and our upper bound techniques are not useful in this setting; essentially $n$ spatial degrees of freedom can be generated by close-by nodes. On the other hand, the multihop communication strategy is interference-limited and the performance scales with area. More precisely, for a fixed area, the upper bound using the cut-set technique yields a scaling law of order $n \log n$ while the multi-hop communication yields a scaling law of order $\sqrt{n}$. Resolving this huge gap, either by careful upper bound techniques or by cleverer communication strategies, remains an open problem.

\section{APPENDIX I}

\section{Optimality of Diagonal COVARIANCE MATRICES}

$\mathbf{H} \in \mathbb{C}^{n \times m}$ is a random matrix with mutually independent entries (i.e., the real and imaginary parts of the entries of $\mathbf{H}$ are all independent). Further, we have that the marginal distribution of each of these random variables is symmetric around the origin. Then consider the following claim:

$$
\begin{aligned}
\max _{\substack{\mathbf{K}_{x} \succeq 0: \\
\left(\mathbf{K}_{x}\right)_{i i} \leq P_{i}}} \mathbb{E}_{\mathbf{H}}\left[\log \operatorname{det}\left(\mathbf{I}+\mathbf{H K}_{x} \mathbf{H}^{\dagger}\right)\right] & = \\
& \mathbb{E}_{\mathbf{H}}\left[\log \operatorname{det}\left(\mathbf{I}+\mathbf{H P H}^{\dagger}\right)\right]
\end{aligned}
$$

Here we have written $\mathbf{P}$ as the diagonal matrix with the $i^{\text {th }}$ diagonal element equal to $P_{i}$. The function being maximized is strictly concave and the set over which it is maximized is convex and compact. Thus the maximization is attained and the maximal value is characterized by the Kuhn-Tucker conditions. The Lagrangian for this problem is

$$
\begin{aligned}
& \mathbf{L}\left(\mathbf{K}_{x}, \mathbf{S}, \mathbf{D}\right)= \\
& \quad \mathbb{E}\left[\log \operatorname{det}\left(\mathbf{I}+\mathbf{H} \mathbf{K}_{x} \mathbf{H}^{\dagger}\right)\right]+\operatorname{tr}\left(\mathbf{S} \mathbf{K}_{x}\right)+\operatorname{tr}\left(\mathbf{D}\left(\mathbf{I}-\mathbf{K}_{x}\right)\right) .
\end{aligned}
$$

The Lagrange variables for the diagonal and positive definite constraints on the input are the nonnegative diagonal matrix $\mathbf{D}$ and positive semidefinite $\mathbf{S}$, respectively. The Kuhn-Tucker conditions for the optimality of $\mathbf{K}_{x}^{*}$ are

$$
\left(\mathbf{K}_{x}\right)_{i i}=P_{i}, \quad \text { and } \frac{d \mathbf{L}\left(\mathbf{K}_{x}, \mathbf{S}, \mathbf{D}\right)}{d \mathbf{K}_{x}}\left(\mathbf{K}_{x}^{*}\right)=\mathbf{0} .
$$

The derivative constraint can be rewritten as

$$
\begin{aligned}
& \frac{d \mathbf{L}\left(\mathbf{K}_{x}, \mathbf{S}, \mathbf{D}\right)}{d \mathbf{K}_{x}}\left(\mathbf{K}_{x}^{*}\right)= \\
& \mathbf{H}^{\dagger}\left(\mathbf{I}+\mathbf{H} \mathbf{K}_{x}^{*} \mathbf{H}^{\dagger}\right)^{-1} \mathbf{H}+\mathbf{S}-\mathbf{D}=\mathbf{0}
\end{aligned}
$$

Our main observation is that

$$
\mathbf{H}^{\dagger}\left(\mathbf{I}+\mathbf{H P H}^{\dagger}\right)^{-1} \mathbf{H}
$$

is a nonnegative diagonal matrix. If we can show this, then we are done: the choice $\mathbf{S}=0$ and $\mathbf{D}$ to the nonnegative diagonal matrix in (37) satisfies the Kuhn-Tucker conditions in (36) with $\mathbf{K}_{x}^{*}=\mathbf{P}$. Consider the off diagonal $(i, j)^{t h}$ element $(i \neq j)$ of the matrix in (37), writing the $i^{t h}$ column of $\mathbf{H}$ by $\mathbf{h}_{i}$ :

$$
\mathbf{h}_{j}^{\dagger}\left(\mathbf{I}+\mathbf{H} \mathbf{P} \mathbf{H}^{\dagger}\right)^{-1} \mathbf{h}_{i}=\frac{\mathbf{h}_{j}^{\dagger} \mathbf{A} \mathbf{h}_{i}}{\left(1+P_{j} \mathbf{h}_{j}^{\dagger} \mathbf{A} \mathbf{h}_{j}\right)\left(1+P_{i} \mathbf{h}_{i}^{\dagger} \mathbf{A} \mathbf{h}_{i}\right)} .
$$

Here we have written $\mathbf{A}:=\left(\mathbf{I}+\sum_{k \neq i, j} P_{k} \mathbf{h}_{k} \mathbf{h}_{k}^{*}\right)^{-1}$ and used the matrix inversion lemma: $\left(\mathbf{B}+\mathbf{x x}^{\dagger}\right)^{-1}=\mathbf{B}^{-1}-$ $\frac{\mathbf{B}^{-1} \mathbf{x} \mathbf{x}^{\dagger} \mathbf{B}^{-1}}{1+\mathbf{x}^{\dagger} \mathbf{B}^{-1} \mathbf{x}}$ whenever the terms exist. Now observe that $\mathbf{A}, \mathbf{h}_{i}, \mathbf{h}_{j}$ are all independent. Thus conditioned on $\mathbf{A}$, we can use the symmetry of the distributions of the elements of $\mathbf{h}_{i}, \mathbf{h}_{j}$ around the origin to claim that the right-hand side of (38) is equal to zero. This justifies the claim in (35).

We can also make a related statement about the optimality of diagonal covariance matrices for the no CSI capacity with an overall power constraint. In particular, consider the following claim (analogous to (35)):

$$
\begin{gathered}
\max _{\substack{\mathbf{K}_{x} \succ 0: \\
\operatorname{tr}\left[\mathbf{K}_{x}\right] \leq P}} \mathbb{E}_{\mathbf{H}}\left[\log \operatorname{det}\left(\mathbf{I}+\mathbf{H K}_{x} \mathbf{H}^{\dagger}\right)\right]= \\
\max _{\substack{\mathbf{D} \succeq 0: \\
\operatorname{tr}[\mathbf{D}] \leq P}} \mathbb{E}_{\mathbf{H}}\left[\log \operatorname{det}\left(\mathbf{I}+\mathbf{H D H} \mathbf{H}^{\dagger}\right)\right]
\end{gathered}
$$

In other words, we can restrict the space of covariance matrices from the cone of positive semidefinite matrices to the cone of nonnegative diagonal matrices ( $\mathbf{D}$ in (39) denotes a diagonal matrix). The proof of (39) follows in much the same way we showed (35). The key component of the proof is to show that with a diagonal $\mathbf{K}_{x}$, the Kuhn-Tucker conditions reduce to those where the input space was already restricted to be diagonal matrices.

\section{REFERENCES}

[1] T. M. Cover, A. A. El Gamal, "Capacity theorems for the relay channel", IEEE Trans. Info. Theory, vol. IT-25, pp. 572-584, Sept 1979.

[2] T. M. Cover and J. Thomas, Elements of Information Theory, John Wiley, 1991.

[3] M. Franceschetti, J. Bruck, and L. Schulman, "Microcellular systems, random walks and wave propagation.", in Proceedings of the IEEE Antennas and Propagation Society International Symposium, 2002., Volume: 1, 16-21 June 2002

[4] P. Gupta and P. R. Kumar, "Capacity of wireless networks", IEEE Trans. on Information Theory, Vol. 46(2), pp. 388-401, Mar 2000.

[5] S.A Jafar and A. Goldsmith, "Multiple-antenna Capacity in Correlated Rayleigh Fading with Channel Covariance Information", submitted to IEEE Transactions on Information Theory.

[6] S. R. Kulkarni and P. Viswanath, "A Deterministic Approach to Throughput Scaling in Wireless Networks", to appear in IEEE Trans. on Information Theory.

[7] O. Lévêque and E. Telatar, "Information theoretic upper bounds on the capacity of large extended ad-hoc wireless networks", submitted to IEEE Trans. on Information Theory, Aug 2003.

[8] A. Santalo, "Integral geometry and geometrical probability", Encyclopedia of Mathematics and its applications, Reading MA: Addison Wesley, 1976, Volume 1.

[9] A. M. Tulino, A. Lozano and S. Verdú, "Capacity-achieving input covariance for correlated multi-antenna channels", Forty-first Annual Allerton Conference on communication, control and computing, 2003.

[10] V. V. Veeravalli, Y. Liang and A. M. Sayeed, "Correlated MIMO Rayleigh Fading Channels: Capacity, Optimal Signaling, and Scaling Laws", submitted to IEEE Transactions on Information Theory, September 2003.

[11] E. Visotsky and U. Madhow, "Space-Time Transmit Precoding With Imperfect Feedback", IEEE Transactions on Information Theory, Vol. 47(6), September 2001. 
[12] L-L. Xie and P. R. Kumar, "A Network Information Theory for Wireless Communication: Scaling Laws and Optimal Operation", submitted to IEEE Transactions on Information Theory, Apr 2002.

[13] F. Xue, L-L. Xie and P. R. Kumar, "Wireless networks over fading channels", submitted to IEEE Transactions on Information Theory, Nov. 2003.

Aleksandar Jovičić received the B.S. in Electrical Engineering from the University of Wisconsin-Madison in 2001 and the M.S. in Electrical Engineering from the University of Illinois at Urbana-Champaign in 2003, where he is currently pursuing his Ph.D. degree. His research interests are in wireless networks and multi-user information theory.

Pramod Viswanath (S'98-A'00) received the Ph.D. degree in electrical engineering and computer science from the University of California at Berkeley in 2000 .

He was a member of Technical Staff at Flarion Technologies until August 2001 before joining the Electrical and Computer Engineering Department at the University of Illinois at Urbana-Champaign.

Prof. Viswanath is a recipient of the Eliahu Jury Award from the Electrical Engineering and Computer Science Department of the University of California, Berkeley (2000), the Bernard Friedman Award from the Mathematics Department of the University of California, Berkeley (2000), and the NSF CAREER Award (2003).

Sanjeev R. Kulkarni (M'91, SM'96, F'04) received the B.S. in Mathematics, B.S. in E.E., M.S. in Mathematics from Clarkson University in 1983, 1984 and 1985, respectively, the M.S. degree in E.E from Stanford University in 1985, and the Ph.D. in E.E. from M.I.T. in 1991.

From 1985 to 1991 he was a Member of the Technical Staff at M.I.T. Lincoln Laboratory working on the modeling and processing of laser radar measurements. In the spring of 1986, he was a part-time faculty at the University of Massachusetts, Boston. Since 1991, he has been with Princeton University where he is currently Associate Professor of Electrical Engineering and Associate Dean of Academic Affairs in the School of Engineering and Applied Science. He spent January 1996 as a research fellow at the Australian National University, 1998 with the Susquehanna International Group, and summer 2001 with Flarion Technologies.

Prof. Kulkarni received an ARO Young Investigator Award in 1992, an NSF Young Investigator Award in 1994, and several teaching awards at Princeton University. He has served as an Associate Editor for the IEEE Transactions on Information Theory. Prof. Kulkarni's research interests include statistical pattern recognition, nonparametric estimation, learning and adaptive systems, information theory, wireless networks, and image/video processing. 